\title{
OPTIMIZATION OF THE CONDITIONS OF CONVECTIVE DRYING OF THERMOSENSITIVE MATERIALS
}

\author{
M.K. AlyzhANOV ${ }^{1}$, M.R. SiKhimbayev ${ }^{2 *}$, S.B. KuZEMBAYEV ${ }^{1}$, \\ K.T. ShEROV ${ }^{1}$, D.R. SiKHIMBAYEVA ${ }^{2}$, T.A. KHANOV ${ }^{2}$, \\ T.B. KurmangAliYeV ${ }^{3}$, D.E. Elemes ${ }^{3}$, B.S. DONENBAYEV ${ }^{1}$, \\ M.M. MusAeV ${ }^{1}$, T.M. Buzauova ${ }^{1}$, \\ ${ }^{1}$ Karaganda State Technical University, \\ 56 B. Mira St., 100027 Karaganda, Kazakhstan \\ ${ }^{2}$ Karaganda Economic University, \\ 9 Akademicheskaya St., 100009 Karaganda, Kazakhstan \\ ${ }^{3}$ D. Serikbayev East Kazakhstan State Technical University, \\ 69 Protazanova St., 070004 Ust-Kamenogorsk, Kazakhstan
}

[Received 04 October 2015. Accepted 12 December 2016]

\begin{abstract}
The article proposes the solution of the problem with the application of thermal vibrations of heat-carrier flow in processes of convective drying, for the purpose of theoretical determination of optimum conditions of short-term excess of handling temperature, that do not influence the decrease of technological characteristics of the thermosensitive materials.
\end{abstract}

KEY WORDS: Optimization, convective drying, thermosensitive materials, heatcarrier flow, optimum temperature, thermal vibrations, controlled parameter, controlling parameter, optimum control.

\section{INTRODUCTION}

In practice of convective drying, the development of regime parameters during handling of thermosensitive materials losing the biological activity at the excess of admissible preheating temperature has special significance. The example can be thermoresponsivity of grain crops (wheat, barley, etc.), which experience a decrease of germination energy of seeds (the basic index of technological properties of a seed pellet), at the excess of optimum temperature of thermal handling (drying). So, the optimum drying temperature of seeds of grain crops, according to various data ranges is within the interval of $60-65^{\circ} \mathrm{C}$. The aforementioned refers to the conditions of unchangeable parameters of a heat-carrier flow used for the process of thermal handling $\left(T_{\mathrm{av}}=\right.$ const $)$.

At the same time, according to the experimental data, short-term excess of optimum temperature by $1.5-2$ times does not deteriorate technological properties of

${ }^{*}$ Corresponding author e-mail: smurat@yandex.ru 
thermosensitive materials [1-3]. Thereupon, an attempt to use pulsing conditions of thermal handling of damp materials with short-term excess (for thermosensitive materials) of admissible drying temperature [4-6], for the purpose to intensify processes of heat-mass exchange is quite justified.

The estimation of influence of flow vibrations of a heat-carrier on the process of convective drying shows that dehumidification of a surface of damp materials is limited not only by a convective component of the process (gas dynamics of the process) and total amount of thermal energy, participating in the process, but also by conditions of the supply of the heat-carrier flow, particular in thermal vibrations [7-9].

\section{STATEMENT OF RESEARCH PROBLEM AND PHYSICAL ESSENCE OF PROCESS}

It is possible to present thermal vibrations as $q_{i}=f(\tau)$, where $q_{i}$ - amount of thermal energy spent for the drying process in $i^{\text {th }}$ period of time, $\tau$ - time of thermal power supply, or as a dependence $\mathrm{d} T / \mathrm{d} \tau=\alpha\left(\mathrm{d} T_{\mathrm{av}} / \mathrm{d} \tau\right)$ [5-6].

$$
q(\tau)=j(\tau)\left[r-c \frac{\mathrm{d} U_{\mathrm{m}}(\tau)}{\mathrm{d} T_{\mathrm{m}}(\tau)}\right]=R_{\mathrm{eq}} \rho_{0} r \frac{\mathrm{d} U_{\mathrm{m}}(\tau)}{\mathrm{d} \tau}\left[1-\frac{c}{r} \frac{\mathrm{d} U_{\mathrm{m}}(\tau)}{\mathrm{d} T_{\mathrm{m}}(\tau)}\right],
$$

where $q$ - amount of thermal energy participating in the drying process; $j-$ sensible heat; $r$ - heat of vaporization; $c$ - thermal capacity; $U_{\mathrm{m}}-$ water content of a material; $T_{\mathrm{m}}-$ material temperature; $R_{\mathrm{eq}}-$ equivalent diameter of material particles; $\rho_{0}$ - material density; $\tau$ - time of supply of thermal energy.

Having entered introducing a relative temperature coefficient of drying $B$, we convert (1) to (2):

$$
q(\tau)=R_{\mathrm{eq}} \rho_{0}\left[r \frac{\Delta U_{\mathrm{m}}}{\Delta T_{\mathrm{m}} * B}+c\right] \frac{\mathrm{d} T_{\mathrm{m}}}{\mathrm{d} \tau},
$$

where $\Delta U_{\mathrm{m}}, \Delta T_{\mathrm{m}}$ - fixed values of water content and temperature.

The dependence between $\ln T_{\mathrm{m}}$ and $B$ (Fig. 1), received experimentally [4-5] by results of drying of various materials in the conditions of thermal and gas-dynamic pulsations of the heat-carrier flow, is close to linear one which will be recorded follows:

$$
\frac{\mathrm{d} \ln T_{\mathrm{m}}}{\mathrm{d} B}=m .
$$

Or

$$
T_{\mathrm{m}}=k_{1} \mathrm{e}^{m B},
$$


where $k_{1}$ - a constant of integration, $m-$ a constant defined only by the type of material and not dependent on drying condition. The coefficient $m$ is defined experimentally for various conditions of supply of the heat-carrier flow [4-6] from the dependence

$$
T_{\mathrm{m}}=f(U) .
$$

The received dependence $B_{1}=\mathrm{d} T_{\mathrm{m}} / \mathrm{d} U$ allows calculating a relative temperature coefficient of drying

$$
B=B_{1} \frac{\Delta U}{\Delta T_{\mathrm{m}}} .
$$

Having presented the results of calculations in a graph $\ln T_{\mathrm{m}}=f(B)$ (Fig. 1), we define a required value of coefficient $(m)$, equal to a tangent of the angle of curve slope $(\alpha)$.

Since the heat, that is necessary to heat a damp material, is in proportion to the difference between temperatures of the environment and a material, then

$$
g(\tau)=\alpha\left[T_{\mathrm{av}}-T_{\mathrm{m}}(\tau)\right] .
$$

Combining (2) and (7), we have:

$$
\alpha\left(T_{\mathrm{av}}-T_{\mathrm{m}}\right)=R_{\mathrm{eq}} \rho_{0}\left[r \frac{\Delta U_{\mathrm{m}}}{\Delta T_{\mathrm{m}} B}+c\right] \frac{\mathrm{d} T_{\mathrm{m}}}{\mathrm{d} \tau} .
$$

Let us present the required problem as a type of an optimum control problem

$$
\frac{\mathrm{d} T_{\mathrm{m}}}{\mathrm{d} \tau}=\frac{\alpha}{R_{\text {eq }} \rho_{0}} \frac{T_{\mathrm{av}}-T_{\mathrm{m}}}{\left[r \frac{\Delta U_{\mathrm{m}}}{\Delta T_{\mathrm{m}} \ln \frac{T_{\mathrm{m}}}{k_{1}}}+c\right]} .
$$

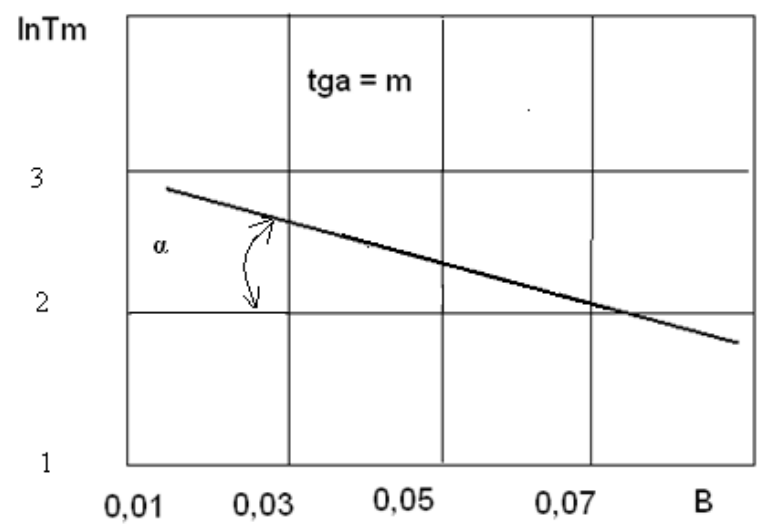

Fig. 1. Dependence $\ln T_{\mathrm{m}}=f(B)$ to define coefficient $m$. 
The controlled parameter here is the temperature of the material $T_{\mathrm{m}}$, and the controlling one - heat-carrier temperature $T_{\mathrm{av}}$.

As an amount of heat, at the persistence of expenditure of the heat-carrier, will be defined by a temperature $\left(T_{\mathrm{av}}\right)$ and sensible heat of material by temperature $\left(T_{\mathrm{m}}\right)$, then considering the problem of maximum use of thermal power of the heat-carrier, we will select the following functional, as a criterion of optimization:

$$
J=\int_{0}^{\tau^{*}} \frac{T_{\mathrm{av}}(\tau)}{T_{\mathrm{m}}(\tau)} \mathrm{d} \tau \rightarrow \inf ,
$$

where the controlling impact is limited to

$$
T_{\mathrm{av}}^{1} \leq T_{\mathrm{av}} \leq T_{\mathrm{av}}^{2} .
$$

Thus, the optimization problem can be formulated as follows: it is required to discover the distribution $T_{\mathrm{av}}(\tau)$, which transfers an object described by the coupling equation (9) from the initial state $T_{\mathrm{m}}(0)=\tau_{\mathrm{m}}^{0}$ to the final one $T_{\mathrm{m}}\left(\tau^{*}\right)=T_{\mathrm{m}}^{k}$. The maximum value of functional $J$ is thus ensured, and the condition (11) is satisfied.

For the problem solution we use the method of maximum principle [10]. Introducing the Lagrange function for this problem

$$
\begin{aligned}
y=\int_{0}^{\tau^{*}}\left[\lambda_{0} \frac{T_{\mathrm{av}}}{T_{\mathrm{m}}}+\mathrm{P}\left(T_{\mathrm{m}}-\frac{\alpha}{R_{\mathrm{eq}} \rho_{0}} \frac{T_{\mathrm{av}}-T_{\mathrm{m}}}{\frac{r \Delta U_{\mathrm{m}}}{\Delta T_{\mathrm{m}} \ln \frac{T_{\mathrm{m}}}{k_{1}}}+c}\right) \mathrm{d} \tau\right] \\
+\lambda_{1}\left[T_{\mathrm{m}}(0)-T_{\mathrm{m}}^{0}\right]+\lambda_{2}\left[T_{\mathrm{m}}\left(\tau^{*}-T_{\mathrm{m}}^{k}\right]\right.
\end{aligned}
$$

where $\tau^{*}$ - time of drying.

The conditions necessary for the process optimization - are the stability of indexes on temperature for the Lagrange function

$$
=\lambda_{0} \frac{T_{\mathrm{av}}}{T_{\mathrm{m}}}+\mathrm{P}\left[T_{\mathrm{m}}-\frac{\alpha}{R_{\mathrm{eq}} \rho_{0}} \frac{T_{\mathrm{av}}-T_{\mathrm{m}}}{\frac{r \Delta U_{\mathrm{m}}}{\Delta T_{\mathrm{m}} \ln \frac{T_{\mathrm{m}}}{k_{1}}}+c}\right]-\frac{\mathrm{d}}{\mathrm{d} \tau} \dot{L}_{1 m}+\dot{L} T_{\mathrm{m}}=0 .
$$

Hence, the interfaced equation in relation to $\mathrm{P}$ is as follows:

$$
=\lambda_{0} \frac{T_{\mathrm{av}}}{T_{\mathrm{m}}}-\mathrm{P} \frac{\alpha}{R_{\mathrm{eq}} \rho_{0}} \frac{\left(\frac{r \Delta U_{\mathrm{m}}}{\Delta T_{\mathrm{m}} \ln \frac{T_{\mathrm{m}}}{k_{1}}+c}\right)+\left(T_{\mathrm{av}}-T_{\mathrm{m}}\right) \frac{r \Delta U_{\mathrm{m}}}{\Delta T_{\mathrm{m}}\left(\ln \frac{T_{\mathrm{m}}}{k_{1}}\right)^{2}} \frac{1}{T_{\mathrm{m}}}}{\left(\frac{r \Delta U_{\mathrm{m}}}{\Delta T_{\mathrm{m}}} \ln \frac{T_{\mathrm{m}}}{k_{1}}+c\right)\left(\frac{r \Delta U_{\mathrm{m}}}{\Delta T_{\mathrm{m}}} \ln \frac{T_{\mathrm{m}}}{k_{1}}+c\right)} .
$$

Optimality on parameter 
$\min \left\{\lambda_{0} \frac{T_{\mathrm{av}}}{T_{\mathrm{m}}}-\mathrm{P} \frac{\alpha}{R_{\mathrm{eq}} \rho_{0}} \frac{T_{\mathrm{av}}}{\frac{r \Delta U_{\mathrm{m}}}{\Delta T_{\mathrm{m}} \ln \frac{T_{\mathrm{m}}}{k_{1}}+c}}\right\}=\lambda \frac{T_{\mathrm{av}}}{T_{\mathrm{m}}}-\mathrm{P} \frac{\alpha}{R_{\mathrm{eq}} \rho_{0}} \frac{T_{\mathrm{av}}}{\left[\frac{r \Delta U_{\mathrm{m}}}{\Delta T_{\mathrm{m}} \ln \frac{T_{\mathrm{m}}}{k_{1}}+c}\right]}$

i.e. optimum control under the condition:

$$
\frac{\lambda_{0}}{T_{\mathrm{m}}}-\mathrm{P} \frac{\alpha}{R_{\mathrm{eq}} \rho_{0}} \frac{1}{\frac{r \Delta U_{\mathrm{m}}}{\Delta T_{\mathrm{m}} \ln \frac{T_{\mathrm{m}}}{k_{1}}+c}} \neq 0
$$

is as follows:

$$
T_{\mathrm{av}}=\frac{T_{2}-T_{1}}{2} \operatorname{sign}\left[\frac{\lambda_{0}}{T_{\mathrm{m}}}-\mathrm{P} \frac{\alpha}{R_{\mathrm{eq}} \rho_{0}} \frac{1}{\frac{r \Delta U_{\mathrm{m}}}{\Delta T_{\mathrm{m}} \ln \frac{T_{\mathrm{m}}}{k_{1}}+c}}\right]+\frac{T_{1}+T_{2}}{2}
$$

In our case we consider the control problem on sites where

$$
\frac{\lambda_{0}}{T_{\mathrm{m}}}-\mathrm{P} \frac{\alpha}{R_{\mathrm{eq}} \rho_{0}} \frac{1}{\frac{r \Delta U_{\mathrm{m}}}{\Delta T_{\mathrm{m}} \ln \frac{T_{\mathrm{m}}}{k_{1}}+c}}=0 .
$$

It is impossible to define the optimum control on the basis of a maximum principle, therefore an additional problem research is required.

Since the function:

$$
\frac{\lambda_{0}}{T_{\mathrm{m}}}-\mathrm{P} \frac{\alpha}{R_{\mathrm{eq}} \rho_{0}} \frac{1}{\frac{r \Delta U_{\mathrm{m}}}{\Delta T_{\mathrm{m}} \ln \frac{T_{\mathrm{m}}}{k_{1}}+c}}
$$

is invariant in relation to the whole space

$$
\begin{gathered}
\frac{\mathrm{d}}{\mathrm{d} \tau}\left(\frac{\lambda_{0}}{T_{\mathrm{m}}}-\mathrm{P} \frac{\alpha}{R_{\mathrm{eq}} \rho_{0}} \frac{1}{\frac{r \Delta U_{\mathrm{m}}}{\Delta T_{\mathrm{m}} \ln \frac{T_{\mathrm{m}}}{k_{1}}+c}}\right)=-\frac{\lambda_{0}}{T_{\mathrm{m}}^{2}} \frac{\mathrm{d} T_{\mathrm{m}}}{\mathrm{d} \tau}-\mathrm{P}\left(\frac{\alpha}{R_{\mathrm{eq}} \rho_{0}}\right)^{2} \frac{T_{\mathrm{av}}-T_{\mathrm{m}}}{\left(\frac{r \Delta U_{\mathrm{m}}}{\Delta T_{\mathrm{m}} \ln \frac{T_{\mathrm{m}}}{k_{1}}+c}\right)^{2} T_{\mathrm{m}}} \\
+\mathrm{P}\left(\frac{\alpha}{R_{\mathrm{eq}} \rho_{0}}\right)^{2} \frac{-\left(\frac{r \Delta U_{\mathrm{m}}}{\Delta T_{\mathrm{m}} \ln \frac{T_{\mathrm{m}}}{k_{1}}+c}\right)}{\left(\frac{r \Delta U_{\mathrm{m}}}{\Delta T_{\mathrm{m}} \ln \frac{T_{\mathrm{m}}}{k_{1}}+c}\right)^{3}}+\mathrm{P}\left(\frac{\alpha}{R_{\mathrm{eq}} \rho_{0}}\right)^{2} \frac{U}{\left(\frac{r \Delta U_{\mathrm{m}}}{\Delta T_{\mathrm{m}} \ln \frac{T_{\mathrm{m}}}{k_{1}}+c}\right)^{2} T_{\mathrm{m}}} \\
-\mathrm{P}\left(\frac{\alpha}{R_{\mathrm{eq}} \rho_{0}}\right)^{2}\left\{\frac{T_{\mathrm{av}}-T_{\mathrm{m}}}{\left(\frac{r \Delta U_{\mathrm{m}}}{\Delta T_{\mathrm{m}} \ln \frac{T_{\mathrm{m}}}{k_{1}}+c}\right)^{3}}-\frac{T_{\mathrm{av}}-T_{\mathrm{m}}}{\left(\frac{r \Delta U_{\mathrm{m}}}{\Delta T_{\mathrm{m}} \ln \frac{T_{\mathrm{m}}}{k_{1}}+c}\right)^{3}}\right\} \equiv 0 .
\end{gathered}
$$




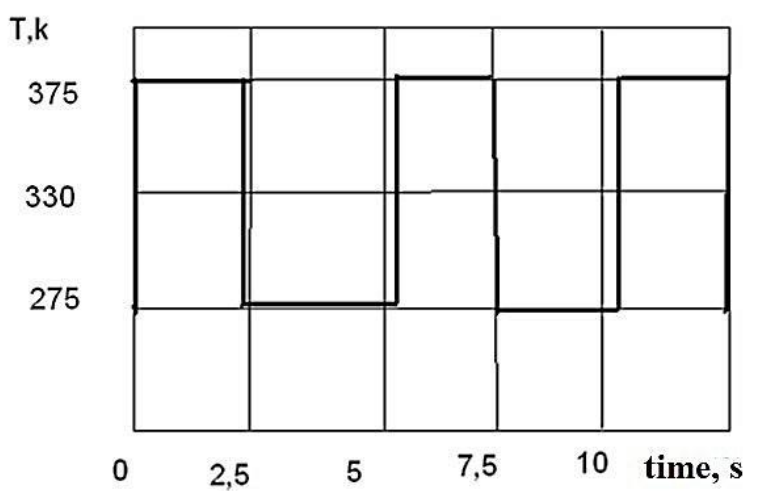

Fig. 2. Optimization of thermal vibrations for convective drying process under conditions that $T_{\mathrm{m}}^{0}=293 \mathrm{~K}, T_{\mathrm{m}}^{1}=330 \mathrm{~K}, T \in(275,375) \mathrm{K}$.

Then, a special extreme can be arbitrarily set on variety (15) and, if we accept it as equal to some fixed value $B$, it follows that each optimum control av $(\tau), 0 \leq \tau \leq \tau^{*}$ is a piecewise constant function, accepting values $T_{1}, T_{2}$ and having not less than two intervals of constancy. Points of switch and interfaces av $(\tau)$ are found from the condition of function continuity $T_{\mathrm{m}}(\tau)$.

As an example, we have carried out the calculation of the optimum temperature control of the heat-carrier flow at convective drying of seeds of wheat of sort "Saratov 29 ", for the experimental data given in [1-2]. In the interval of 275-385 K, according to the drawing (Fig. 2). For experimental conditions, the time of presence of the material particles in the zone of the thermal flow impact (time of drying $\tau^{*}$ ), corresponded to 5.2 seconds. During the solution of the specified problem, a graph of heat-carrier temperature change, which satisfied the process of convective drying, was obtained.

For conditions, when the temperature of the heat-carrier flow is maintained as stable throughout the process (absence of thermal vibrations), the optimum temperature of drying of wheat seeds, ensuring the preservation of their technological properties (energy of germination) [1-2], is within the range of $325 \div 335 \mathrm{~K}$ (Fig. 3). When lowering of the heat-carrier flow temperature, used for the process, efficiency and pace of drying machines decrease. Accordingly, the time of drying increases and the efficiency of the drying machines decreases. At the same time, at excess of the marked range of temperatures of the heat-carrier flow, as the experimental data [1-2] have been displayed, the energy of germination of seeds sharply decreases. Excess of optimum temperature during of thermosensitive materials leads to deterioration of their technological properties, lowering the quality and faults.

The use of thermal vibrations of the heat-carrier flow with short-term excesses and the decrease of the optimum range $(325 \div 335 \mathrm{~K})$ within \pm 50 degrees did not 


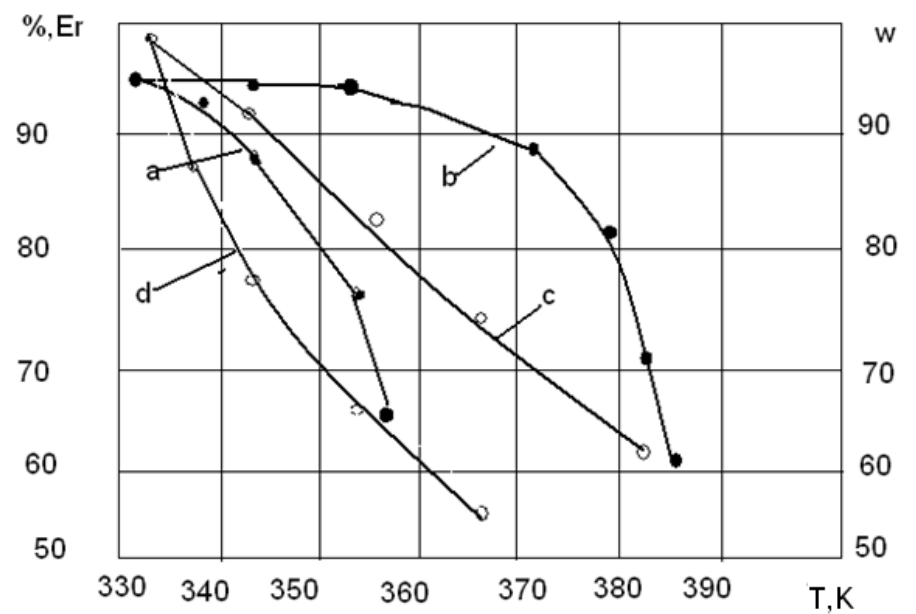

Fig. 3. Dependence of energy of germination $(\%, \mathrm{Er})$ and dehumidification degree $(\%, \mathrm{w})$ on process temperature $(\mathrm{T}, \mathrm{K})$, at convective drying of wheat seeds where: a-dependence of energy of germination (Er) on the process temperature $(\mathrm{T})$ for a traditional (non-thermal vibrations) condition of drying; $\mathbf{b}$ - dependence of energy of germination (Er) on process temperature $(\mathrm{T})$ for condition of thermal vibrations; $\mathbf{d}$ - dependence of dehumidification degree $(\mathrm{w})$ on process temperature $(\mathrm{T})$ for a traditional (non thermal vibrations) condition of drying; c - dependence of dehumidification degree (w) on process temperature $(\mathrm{T})$ for condition of thermal vibrations.

influence lowering of energy of germination of seeds, i. e. deterioration of technological properties of thermosensitive materials [1-2]. Thus, for a condition of thermal vibrations of the heat-carrier flow, the temperature range of convective drying process with a reference to the examined material has essentially expanded.

The received experimental data confirm the competence of the use of pulsing conditions at convective drying of thermosensitive materials. Thermal vibrations provide the essential expansion of a range of the temperatures used in the process with the preservation of technological properties of a material (curves a and b) and it also allows simultaneous growth of the degree of drying of a material, at smaller expenditures of thermal energy (curves $d$ and $c$ ).

\section{CONCLUSiOnS}

1. The solution of an optimization problem of convective drying allows defining admissible oscillations of temperature of the heat-carrier flow $\left(T_{\mathrm{av}}\right)$ with constraint of a preheating temperature value of a material $\left(T_{\mathrm{m}}\right)$, that in turn creates preconditions for the control of drying process of thermosensitive materials, at short-term excesses of admissible temperature of their heat with nondeterioration of technological properties. 
2. The offered solution of the optimization problem, with an application in practice will allow us essentially to expand the temperature range of the heat-carrier flow in processes of convective drying of thermosensitive materials.

3. The use of thermal vibrations in convective drying conditions will enable to intensify processes of heat-mass exchange and, thereby, to enhance the capacity of industrial dryers.

4. The use of thermal pulsing conditions will enable us to control the phases of interacting at thermal (drying) or thermochemical (redox reactions) processes and at the expense of it, to discover more effective treatment schedules.

5. Pulsing thermal treatment schedules can display their efficiency in processes of thermochemical handling in heterophase environments, such as: redox processes in metallurgical ovens, thermocatalytic processes in petrochemistry etc. It will allow determining new ways of intensification, occurring in metallurgical and chemical aggregates of processes.

6. For conditions of redox processes, it is necessary to select other criteria (density of ambient components, material composition, etc.), as parameters of optimization.

\section{REFERENCES}

[1] Maximov, E. V., B. S. Fialkov, M. K. Alyzhanov. Formation of a Zone of Circulation in a Dense Bed at Pulse Supply of Gas. Magazine of News of Higher Education Institutions, Ferrous Metallurgy, 3 (1984), 32-33.

[2] Maximov, E. V., B. S. Fialkov, M. K. Alyzhanov. Processes of drying of Aglodomenny Raw Materials in the Pulsing Mode, Automation and Mechanization of Technological Processes of Production in Ferrous Metallurgy of Kazakhstan, Karaganda, Chermetavtomatika, 1984, 65-66.

[3] Maximov, E. V., M. K. Alyzhanov, M. M. Bukenov, B. S. Fialkov. Matematicheskaya Model of Interaction of a Gas Stream with a Dense Bed of Bulk, VINITI, 10.01.1986, No. 228-B86. The Deposited Manuscript, 1986.

[4] Alyzhanov, M. K., E. V. Maksimov, Sn. K. Kapbasov. Dewatering and drying of Iron - Ore Concentrates in the Regime of Active Interation Turbulent Gas Flow, Metallurgu - 92, Modernization of Iron and Itell Industry and New Processes of Steel Production, Cracow, 1992.

[5] Alyzhanov, M. K., D. E. Dusembaev, V. G. Kim. Large - scale Formations of Concentrated Dispersion Mediums Inshift Flows, Chissa - 93, Praga, 1993.

[6] Alyzhanov, M. K. The Moisture in the Pulsatile Mode, Proceedings of the University of Karaganda, KSTU, 2004, No.1, 52-54. 
[7] LyKov, A. V., P. S. Kuts, L. S. Slobodkin. An Approximate Method for Calculating the Kinetics of the Drying Process. Journal of Engineering Physics, 8 (1967), No. 5, 112-119.

[8] Vyrubov, D. N., ET AL. The Internal Combustion Engines, Theory Piston and Combined Engines, Moscow, Mashinostroenie, 1983.

[9] Atanov, G. H., A. I. Petrakov. Impulsive Hydrodynamic Method of Rock Breaking, Proc. Int. Conf. Eros. Liquid and Solids Import, Cambridge, 5-8 Sept. 1983, s. a. 32/1-32/8.

[10] Alekseev, V. M., V. M. Tikhomirov, S.V. Fomin. Optimal Control, Moscow, Science, 1979. 